\title{
Study of nuclear tracks on different polycarbonate foils and their feasibility for use in personnel monitoring
}

\author{
V Jayalaskmi*, Rupali Rohatgi, Deepa Sathian, P K Marathe, Sarala Nair, \\ G Chourasiya and S Kannan \\ Radiological Physics and Advisory Division, Bhabha Atomic Research Centre, Mumbai-400 085, India \\ E-mail : j_van@indiatimes.com
}

\begin{abstract}
CR-39 (PADC) a Solid State Nuclear Track Detector., is used as a routine personnel monitor. 1800 workers are being monitored quarterly for fast neutron using CR-39 foils. These foils procured from Pershore Mouldings, UK are very expensive and indigenous development will make the foils cost effective. The aim of this paper is to find a suitable alternative to the imported CR-39 foils for use in personnel monitoring. The foils from three different manufacturers have been compared with the CR-39 foils from Pershore Moulding, UK, presently in use. Out of the three, only sample no 1 is promising. It has a background and sensitivity comparable with CR-39 presently used. The sample 2 is CR-39 being developed in India, has a relatively high background and poor sensitivity. Efforts are being made to improve the quality of this sample. The sample 3 was a poly carbonate from local manufacturer which produced very few tracks and the standard deviation of track counts was very large and hence not useful for Personnel Monitoring.
\end{abstract}

Keywords : CR-39- PADC, polycarbonate, sensitivity, background.

PACS Nos. : 29.40.-O, 87.53.Bn, 81.65.Cf

\section{Introduction}

Solid State Nuclear Track Detection (SSNTD) [1,2] is the technique of permanently recording the tracks of ionizing particles in insulating solids and was discovered by Young in 1958. Since then, many insulating materials have been tested and plastic material was found to be the most useful due to the ease with which the nuclear tracks could be revealed by simple method of chemical etching and by viewing them under microscope. Currently, CR-39 foils made of poly-allyl diglycol carbonate polymer (PADC) (Figure 1) are mainly used for assessing neutron personnel dose equivalent at the work place. CR-39 (PADC) has the advantages that it offers a high response to protons, it is insensitive to beta and gamma radiation up to $50 \mathrm{~Sv}$, there is no post irradiation fading of damaged tracks in extreme conditions of temperature and humidity and counting is easy. With appropriate use of materials with high hydrogen content as 
neutron-proton 'converters/ radiators', a flat dose equivalent response for neutrons from $100 \mathrm{keV}$ to $10 \mathrm{MeV}$ can be achieved.

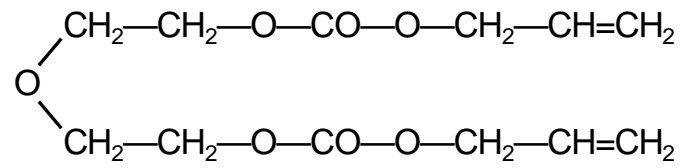

Figure 1. Chemical formula for PADC.

In India 1800 radiation workers are being monitored routinely using fast neutron personnel monitoring badges [3]. The fast neutron badge comprises of CR-39 foil with a $1 \mathrm{~mm}$ polyethylene radiator in front sealed in a triple laminated aluminized pouch and a badge holder. Radiator serves the purpose of enhancing sensitivity for track detection due to recoil proton, protecting the foil from environmental radiation, and to make it dust proof during handling. CR-39 foils of dosimetric grade are presently imported from Pershore Mouldings, UK and are very expensive and hence the need for development of CR-39 foils indigenously. The aim of this paper is to find a cost effective alternative to the imported CR-39 foils for use in personnel monitoring. A comparison of the background and the neutron dose equivalent response of plastics made by different manufacturers are presented in this paper.

\section{Materials and methods}

Samples of polycarbonates were procured from three different manufacturers. The samples included SAMPLE 1 : CR-39 imported from Germany, SAMPLE 2 : indigenously developed CR-39 also a PADC, which is presently being used for detection of alpha and fission fragments, and SAMPLE 3 : locally available polycarbonates having chemical composition $\mathrm{C}_{16} \mathrm{H}_{14} \mathrm{O}_{3}$. The response of the CR-39 samples such as the background and sensitivity, were compared with response of CR-39 PADC from Pershore Mouldings, UK, having chemical composition $\mathrm{C}_{12} \mathrm{H}_{18} \mathrm{O}_{7}$ presently in use. The thickness of each detector was first measured after removing the protective layers. The thicknesses of sheets varied from $600 \mu \mathrm{m}$ to $800 \mu \mathrm{m}$.

Sheets were cut into $3 \mathrm{~cm} \times 3 \mathrm{~cm}$ size and sealed in triple laminated pouches (having a thin layer of aluminum, paper and polyethylene) with $1 \mathrm{~mm}$ polyethylene radiator of same dimension in front of the foil, and used in these studies. The background was measured on either sides of the foil to identify the 'front side' or the side with higher sensitivity. In case of sheets from Pershore Mouldings background was same on both sides.

\subsection{Irradiation of foils :}

Ten samples, of foils taken from sheets supplied by each of the three manufacturers along with the radiator in front of each foil, was exposed to $1 \mathrm{mSv}$ neutron dose from 
$1 \mathrm{Ci} \mathrm{Am-Be} \mathrm{source,} \mathrm{and} \mathrm{an} \mathrm{equal} \mathrm{number} \mathrm{of} \mathrm{samples} \mathrm{served} \mathrm{as} \mathrm{control} \mathrm{foils.} \mathrm{The}$ irradiations were performed in a laboratory under low scatter conditions. The foils were irradiated at a distance of $50 \mathrm{~cm}$. in air, for $8 \mathrm{hrs}$ and 55 mins with the radiator side facing the Am-Be neutron source having neutron yield of $2.5 \times 10^{6} \mathrm{n} / \mathrm{sec}$.

\subsection{Processing of foils :}

The processing of all the exposed as well as unexposed foils, is carried out in two step electrochemical process at an Elevated Temperature Electro Chemical Etching (ETECE) [3] cell in which 16 foils can be mounted at a time. The ETECE cell is kept in an incubator maintained at $60^{\circ} \mathrm{C}$ during electrochemical etching. At one end of the cell a plane steel plate serves as a neutral electrode, while at the other end, there are electrodes dipped into small cavities filled with $7 \mathrm{~N} \mathrm{KOH}$ solution through which current is allowed to pass. This electrode keeps changing the frequency on the side of the foil, which has been exposed to radiation through a $1 \mathrm{~mm}$ thick polyethylene radiator. In the first stage, the power supply operates at low frequency of $100 \mathrm{~Hz}$ and 1250 volts constant potential for 4 hours, followed by second stage with power supply operating at high frequency of $3.5 \mathrm{KHz}$ for $40 \mathrm{~min}$. The ETECE cell is cooled for $2 \mathrm{hrs}$ and the foils are then washed in running water thoroughly to remove all the alkali solution and dried at room temperature over night.

\subsection{Track counting of foils using image analysis system :}

After processing, the counting of damaged tracks over one square $\mathrm{cm}$ area of each of the foils was carried out using a PC based automatic image analysis system developed in BARC [4] which comprises of a video camera, image frame grabber and suitable light arrangement fixed in a light proof black box having a sliding tray to mount the etched foil for counting. The image analysis system has suitable software which counts the tracks with diameter greater than the one selected for counting, eliminating all other overlapping tracks or deformities which otherwise would have been treated as a track. The control foil is first mounted and number of tracks counted after selection of the track diameter. The soft ware package then scans through the exposed foils for tracks over a designated area of $1 \mathrm{sq} \mathrm{cm}$. The display indicates net tracks counted after subtraction of back ground (control). The net track counted is multiplied by an appropriate factor i.e., $\mathrm{mSv} /$ net track count, which depends on the detector, the neutron source available with the user and the image analysis system, to arrive at the dose equivalent. The average background recorded by samples from different manufacturers is obtained from average counts recorded by 10 unexposed and processed foils. The minimum detectable dose in $\mathrm{mSv}$ for the sample/processing/detector system, has been estimated from the net tracks $\left(\mathrm{cm}^{-1}\right)$ counted for given exposure $(\mathrm{mSv})$ and the standard deviation $\left(\sigma\right.$ in $\left.\mathrm{cm}^{-1}\right)$ of the background counts obtained for 10 unexposed and 
processed foils, using the following relationship :

Dose $(\mathrm{mSv}) \times 3 \sigma$ (background counts) $\left(\mathrm{cm}^{-1}\right)$

Net track counts $\left(\mathrm{cm}^{-1}\right)$

Processing of foils using two-step electrochemical etching enables physical enlargement of the tracks from 10 to 100 micrometers in diameter. Tracks were not accepted as genuine unless their areas and roundness fell within the acceptance criteria. To measure the roundness the formula (perimeter) $)^{2} /\left(4 \pi \times \pi r^{2}\right)$ is used. Roundness of 1 corresponds to a disc. The upper and lower limits for diameter of acceptable tracks were 60 and $400 \mu \mathrm{m}$ and for roundness 0.7 and 1.8 , respectively.

\section{Results and discussion}

The characteristics such as back ground tracks, sensitivity and minimum detectable dose (MDD), of the polycarbonate foils from three different manufacturers have been compared with the CR-39 (PADC) foils from Pershore Moulding, UK, in Table 1.

Table 1. Comparative study of the response of different polycarbonates.

\begin{tabular}{llllll}
\hline $\begin{array}{l}\text { Sample } \\
\text { No. }\end{array}$ & $\begin{array}{l}\text { Thick- } \\
\text { ness } \\
\text { of foil } \\
(\mu \mathrm{m})\end{array}$ & $\begin{array}{l}\text { Ave } \\
\text { Track } \\
\text { diameter } \\
(\mu \mathrm{m})\end{array}$ & $\begin{array}{l}\text { Back- } \\
\text { ground Av. } \\
\text { Counts } \\
\left(\mathrm{cm}^{-2}\right)\end{array}$ & $\begin{array}{l}\text { Exp. to 1 } \\
\text { mSv Ave } \\
\text { Counts } \\
\left(\mathrm{cm}^{-2}\right)\end{array}$ & $\begin{array}{l}\text { MDD } \\
\text { in } \\
\mathrm{mSv}\end{array}$ \\
\hline $\begin{array}{l}\text { CR-39 } \\
\text { (UK) }\end{array}$ & 625 & 90.6 & $42 \pm 7.1$ & $145 \pm 10.6$ & 0.20 \\
Sample 1 & 610 & 105 & $39.16 \pm 12.1$ & $118 \pm 39.26$ & 0.30 \\
Sample 2 & 675 & 100.75 & $117 \pm 12.2$ & $181 \pm 27.15$ & 3.60 \\
Sample 3 & 800 & 383 & $3.6 \pm 0.6$ & $10 \pm 4.5$ & - \\
\hline
\end{tabular}

Out of the three, only the sample 1 : which is a PADC is promising. It has a background and sensitivity comparable with CR-39 presently used, and has good reproducibility. The sample 2 is CR-39 PADC being developed in India, has a relatively high background and poor sensitivity for protons. Efforts are being made to improve the quality of this sample by adopting different methods of production in the lab scale at Department of Chemistry, Goa University [5]. CR-39 sheets having different background and sensitivity are produced by varying the methods of production $[6,7]$. The chemical components of the CR-39 plastic include a monomer, initiator and a plastisicer. Isopropyl peroxide (IPP) is the most commonly used initiator and dioctyl phthalate (DOP) the most used plasticizer. Plasticizer prevents loss of optical clarity (fogging) when dosimeters are chemically etched. Different methods of production lead to different dimer and trimer components which effect the curing. PADC is cured vertically in water baths or horizontally in ovens with temperature being raised according to a specified set of steps. The monomer used for production of sample 2 is PADC. The sample 3 was poly carbonate from local manufacturer which produced very few tracks 
and the standard deviation of track counts was very large and hence not useful for Personnel monitoring. Poly carbonate foils have a fast neutron energy threshold in the range from 1-12 MeV. A significant fraction of the detected secondary particles in most work places are elastically scattered protons. None of the secondary charged particles generated by neutrons in the energy range $100 \mathrm{keV}-1 \mathrm{MeV}$ can produce enough damage in the polycarbonate detector for readable tracks to be produced. This is due to the fact that, PADC has a higher hydrogen content. The maximum energy imparted by elastic scattering for hydrogen is $100 \%$, for carbon $28 \%$ and oxygen $22 \%$ of incident neutron energy. Additionally, the carbon and oxygen recoils have very short ranges due to high LET and low energies, which make their chemically etched tracks difficult to read and also limit the scope for dielectric break down when an electrical field is applied.

However, for high neutron energies the secondary charged particles of low LET may produce etchable tracks during the last 20 micron of their path. At higher energies, nuclear reactions that generate charged particles can also produce tracks in the detector.

\section{Conclusions}

The neutron energies encountered in most work places are in the range of thermal to $1 \mathrm{MeV}$ and hence only CR-39 PADC foils can record tracks due to the secondary charged particles produced by neutrons in the work environment. Out of the CR-39 PADC samples only the sample 1 had a response suitable for personnel monitoring. The sample 2 is in the process of development and sample 3 is not suitable for fast neutron personnel monitoring.

\section{Acknowledgment}

Acknowledgments are due to Dr. V S Nadkarni, Department of Chemistry, Goa University for supplying us with the samples of CR-39 developed in his laboratory for studying its sensitivity to protons.

\section{References}

[1] R J Tanner, D T Bartlett and L G Hager Radiation Measurements 40549 (2005)

[2] J R Harvey and A R Weeks Radiation Protection Dosimetry 2089 (1987)

[3] M P Dhairyawan, P K Marathe and O P Massand Radiation Measurements 36435 (2003)

[4] Y V Rao, S Kar, G Valli, D G Joshi, V K Chaddaa, R K Nigam, OP Massand, M P Dhairyawan and P K Marathe Image Analysis System for Automation in Fast Neutron Personnel Monitoring using CR-39 Solid State Track Detector, International Conference on Automation 1CAUTO-95 (Indore, India) (1995)

[5] A Adelete, A Mascarenhas, S G Tilve and V S Nadkarni Designed Monomers and Polymers 8177 (2005)

[6] J R Harvey, A P French, M Jackson M C Renouf and A R Weeks Radiation Protection Dosimetry 70149 (1997)

[7] R V Griffith, J H ThornGate, K J Davidson, D W Rueppel and J C Fisher Radiation Protection Dosimetry 161 (1981) 